\title{
PHYSICAL PROPERTIES OF THE EXTRANUCLEAR EMITTING REGIONS IN IRREGULAR AND INTERACTING AGNS
}

\author{
P. RAFANELLI \\ Department of Astronomy, University of Padova \\ Padova, Italy
}

\begin{abstract}
CCD slit spectra have been obtained at Lick Observatory of a sample of fourteen close interacting and irregular Seyfert galaxies. The spectra of the extranuclear emitting regions have been investigated and classified using the quantitative criteria recommended by Veilleux and Osterbrock to distinguish objects photoionized by thermal sources from objects photoionized by a "power law" continuum. From our data it results that four out of the twenty emitting regions identified are typical H II regions, while eight have spectra photoionized by a hard-photon spectrum. Seven regions show a twofold behavior which is interpreted in terms of a combination of photoionization, shock heating and scattering of light by dust.
\end{abstract}

\section{Observations}

Long slit spectroscopy of a sample of fourteen close interacting and irregular active galaxies, having redshift $\mathrm{z} \leq 0.06$, was performed to search for extranuclear emitting regions and to study their physical conditions. The spectra were obtained with the CCD lens/grism spectrograph at the Cassegrain focus of the Shane 3-m telescope of the Lick Observatory. All the spectra were taken with a Texas Instruments $800 \times 800$ three-phase CCD and cover the spectral range $\lambda \lambda 4000-7800 \AA$. Their typical resolution is $\sim 18 \AA$. The spectra were calibrated, reduced and measured using the VISTA imageprocessing package, developed at Lick Observatory, and the spectral analysis program RETICENT. The H $\alpha$ and [N II] lines were separated, when they were not resolved, fitting their profile with gaussians.

\section{Results}

No one of the three Seyfert 1 galaxies observed (Mkn 1395, Mkn 885, Kaz 163) shows extranuclear emitting regions. Conversely twenty extended extranuclear emitting regions have been identified in seven out of eleven Seyfert 2s (Mkn 334, E0116+37, Mkn 266, Mkn 273, Mkn298, Mkn 700, NGC 5953). A classification of their spectra, addressed to identify the nature of their ionization source, was performed using the line 
ratios and the diagnostic diagrams proposed by Veilleux and Osterbrock (1987) to distinguish between $\mathrm{H}$ II region-like objects and AGNs. While the [O III $] \lambda 5007 / \mathrm{H} \beta$ versus $[\mathrm{O} \mathrm{I}] \lambda 6300 / \mathrm{H} \alpha$ and $[\mathrm{O} \mathrm{III}] \lambda 5007 / \mathrm{H} \beta$ versus $[\mathrm{S} \mathrm{II}](\lambda 6716+\lambda 6731) / \mathrm{H} \alpha$ intensity ratios show a tendency to cluster in the part of the diagrams accupied by the AGNs, the $[\mathrm{O}$ III] $\lambda 5007 / \mathrm{H} \beta$ versus $[\mathrm{N} \mathrm{II}] \lambda 6583 / \mathrm{H} \alpha$ intensity ratios are almost equally distributed between AGNs and H II region-like objects. This difference in distribution cannot be ascribed to effects produced by errors in the measurement of the line fluxes, since these should introduce in our case only an effect of $\pm 0.1 \mathrm{dex}$ in the line ratios. The only ambiguous case is that of the emitting region to the south-east $\left(P . A .=156^{\circ}\right.$ ) of $\mathrm{Mkn}$ 309 , which is located in all three diagrams on the border between AGNs and H II regions. Excluding this case it results that four out of the twenty identified regions (two in NGC 5953, one in Mkn 309, one in E0116+317) have line ratios consistent with photoionization by a thermal continuum, while eight of them (one in $\mathrm{Mkn} 334$, four in Mkn 266, two in Mkn 273, one in Mkn 700) show line ratios produced by a hardphoton spectrum as in the AGNs. The remaining seven regions ( Mkn 334) show a twofold behavior. Those located in Mkn 298 have [O I] $\lambda 6300 / \mathrm{H} \alpha$ and $[\mathrm{S} \mathrm{II}](\lambda 6716+\lambda 6731) / \mathrm{H} \alpha$ ratios typical of $\mathrm{AGNs}$, while their $[\mathrm{N} \mathrm{II}] \lambda 6583 / \mathrm{H} \alpha$ ratio is as low as in in typical H II regions. A similar situation occurs in Mkn 334 the spectrum of which is characterized by [N II] $\lambda 6583 / \mathrm{H} \alpha$ and [O I] $\lambda 6300 / \mathrm{H} \alpha$ ratios similar to those of AGNs while the $[\mathrm{S} \mathrm{II]}(\lambda 6716+\lambda 6731) / \mathrm{H} \alpha$ ratio suggests the presence of photoionization by a thermal source.

\section{Discussion}

There is no doubt that the regions which were classified without ambiguity belong either to the class of the H II regions or to the class of the objects photoionized by a nonthermal spectrum, possibly the nuclear power-law continuum. An attempt to fit the line ratios of the extranuclear emitting regions located in Mkn 298 and Mkn 334 with the shock models published by Shull and Mc Kee (1979) has been unsuccessful. However it has to be taken in account that the spectra of these regions can be produced by a combination of photoionization by internal thermal sources, photoionization by the nonthermal continuum of the nucleus, ionization by shocks and finally scattering by dust of the light coming from the nucleus. Such a combination can very likely reproduce the anomalous line ratios observed in Mkn 298 and Mkn 334.

\section{References}

Shull, J.M. and McKee, C.F. 1979, Ap. J., 227, 131

Veilleux, S., and Osterbrock, D.E. 1987, Ap. J. Suppl., 63, 295 